\title{
Colorectal Carcinoma in a Patient with Situs Inversus Totalis
}

\author{
lunius Paul Simu¹, Janos Jung², Tivadar Bara ${ }^{3}$, Patricia Maria Luminita Simu4* ${ }^{*}$, Alexandru Ghizdavat ${ }^{5}$ \\ 1. Department of Radiology and Imaging, University of Medicine and Pharmacy Tîrgu Mures, Romania \\ 2. Department of Pathology, University of Medicine and Pharmacy Tirgu Mures, Romania \\ 3. Department of Surgery, University of Medicine and Pharmacy Tirgu Mures, Romania \\ 4. University of Medicine and Pharmacy Tirgu Mures, Romania \\ 5. Department of Anatomy, University of Medicine and Pharmacy Tirgu Mures, Romania
}

Introduction: Colorectal cancer is one of the most common types of malignant tumors worldwide. In patients with situs abnormalities such as situs inversus totalis or situs ambiguus, the presence of this tumor could be a challenge for the surgeon, especially in cases in which the laparoscopic approach is considered. Case presentation: We report the case of a 69-year-old male patient with situs inversus totalis. This particular case of situs inversus totalis was not a classical type because the patient had bilateral bilobed lungs, polysplenia, preduodenal portal vein in association with midgut malrotation. The pathology report after surgery revealed moderately differentiated adenocarcinoma of the sigmoid colon, stage pT3 N1c M1a, liver metastases but without metastases in the eight resected lymph nodes. We compared this rare association of diseases of particular anatomic aspects with other reports in the specialty literature. Conclusion: The identification of situs abnormalities or other malformations in patients with resectable colorectal cancer is essential, thus preoperative imaging studies are imperative for a proper surgical management. Colorectal cancer metastasizing patterns in patients with intestinal malrotation need to be further investigated.

Keywords: situs inversus totalis, colorectal cancer, midgut malrotation, polysplenia, bilateral bilobed lungs

Received 21 November 2017 / Accepted 10 December 2017

\section{Introduction}

Colorectal cancer (CRC) is the third most common type of cancer in males and second in females, which makes it one of the most common causes of death worldwide [1]. Over the past few decades, CRC screening methods improved significantly, hence becoming indispensable, especially for detecting earlier-stage CRC. CRC usually presents less specific or no symptoms at all. The surgical approach is mandatory in all operable stages, hence, in the rare case of a situs abnormality, an accurate imaging evaluation of the anatomy is essential. Situs abnormalities include situs inversus totalis (SIT) and situs ambiguus (SA). SIT is a rare congenital condition, with an incidence of 1:10000 births, in which the arrangement of all internal organs are mirrored, hence a complete transposition of organs [2]. SA (or heterotaxy) is characterized by visceral malposition with the ambiguity of atrial morphology. There are two main categories of SA, double left-sidedness or left-isomerism (polysplenia, bilobed bilaterally lungs) and double rightsidedness or right-isomerism (asplenia, trilobed bilaterally lungs). SA has an incidence of 1:10000 - 1:20000 births [2]. Baso M. et al. have reported a number of 14 associations between SIT and colorectal cancer [3]. In this paper, we report a rare case of sigmoid cancer in a 69-year-old male patient with SIT, polysplenia and intestinal malrotation. The patient signed the informed consent for surgery and the publication of this case before the intervention.

\footnotetext{
* Correspondence to: simupatricia@gmail.com
} E-mail: simupatricia@gmail.com

\section{Case presentation}

A 69 year-old-male patient was admitted to the department of surgery for weight loss, vague abdominal pain and chronic diarrhoea. Personal history included SIT, primary hypertension, obliterating arteriopathy of the lower limbs. Physical examination revealed nothing remarkable except the heart sounds which were heard on the right side of his chest and the dull percussion sound of the liver on the left side of his abdomen. Laboratory findings revealed mild anemia (hemoglobin $12 \mathrm{mg} / \mathrm{dl}$, hematocrit 39\%).

The CT scan revealed situs inversus: the location of the heart on the right side with complete transposition of the heart chambers and the great arteries with normal visceroatrial arrangement, concordant atrioventricular connection and normal emergence of the great arteries (Figure 1). The bilobed lungs were present bilaterally without minor fissure (Figure 2). The suprarenal segment of the inferior vena cava was absent and the azygous vein continuation drained into the superior vena cava. Furthermore, our patient had polysplenia and intestinal malrotation. The portal vein had a preduodenal and prepancreatic course. The ligament of Treitz was present, but the small bowel was situated on the right side of the midline and the colon on the left side of the midline. The superior mesenteric vein was situated on the right side of the superior mesenteric artery. The ileocecal junction was found in the middle of the lower abdomen. There was no fixation of the ascending colon with only one left flexure behind the posterior margin of the liver. The sigmoid colon had the mesocolon on the left part of the pelvis. A moderate circumferential 
thickening of the walls in the sigmoid colon $(13 \mathrm{~mm}$ thick and $30 \mathrm{~mm}$ long), minimal infiltration of the surrounding fat and three perisigmoid lymph nodes up to $6 \mathrm{~mm}$ were observed. Two hypovascular metastases were noticed in the liver. Diffuse atherosclerotic disease was present with multiple calcified and noncalcified plaques. After the assessment, the patient underwent the surgical resection of the tumor and liver metastases using the classical procedure with laparotomy, followed by mechanical colorectal anastomosis. An anastomosis fistula occurred, which required reintervention. The patient was discharged after three weeks of hospitalization. The pathological result revealed the presence of moderately differentiated adenocarcinoma with two hepatic metastases and no involvement of the eighth paracolic resected lymph nodes. Medical imaging suggested $\mathrm{cT}_{3} \mathrm{~N}_{1 \mathrm{~b}} \mathrm{M}_{1 \mathrm{a}}$ stage tumor, after the pathohistological examination the tumor stage was $\mathrm{pT}_{3} \mathrm{~N}_{1 \mathrm{c}} \mathrm{M}_{1 \mathrm{a}}$. Chemotherapy was prescribed which included oxaliplatin $85 \mathrm{mg} / \mathrm{m}^{2}$, leucovorin $500 \mathrm{mg} / \mathrm{m}^{2}$ and 5-fluorouracil 400 $\mathrm{mg} / \mathrm{m}^{2}$ with imaging follow-up in six months from now.

\section{Discussions}

The presence of SIT in a CRC patient could represent a challenge for the surgeon when choosing the laparoscopic

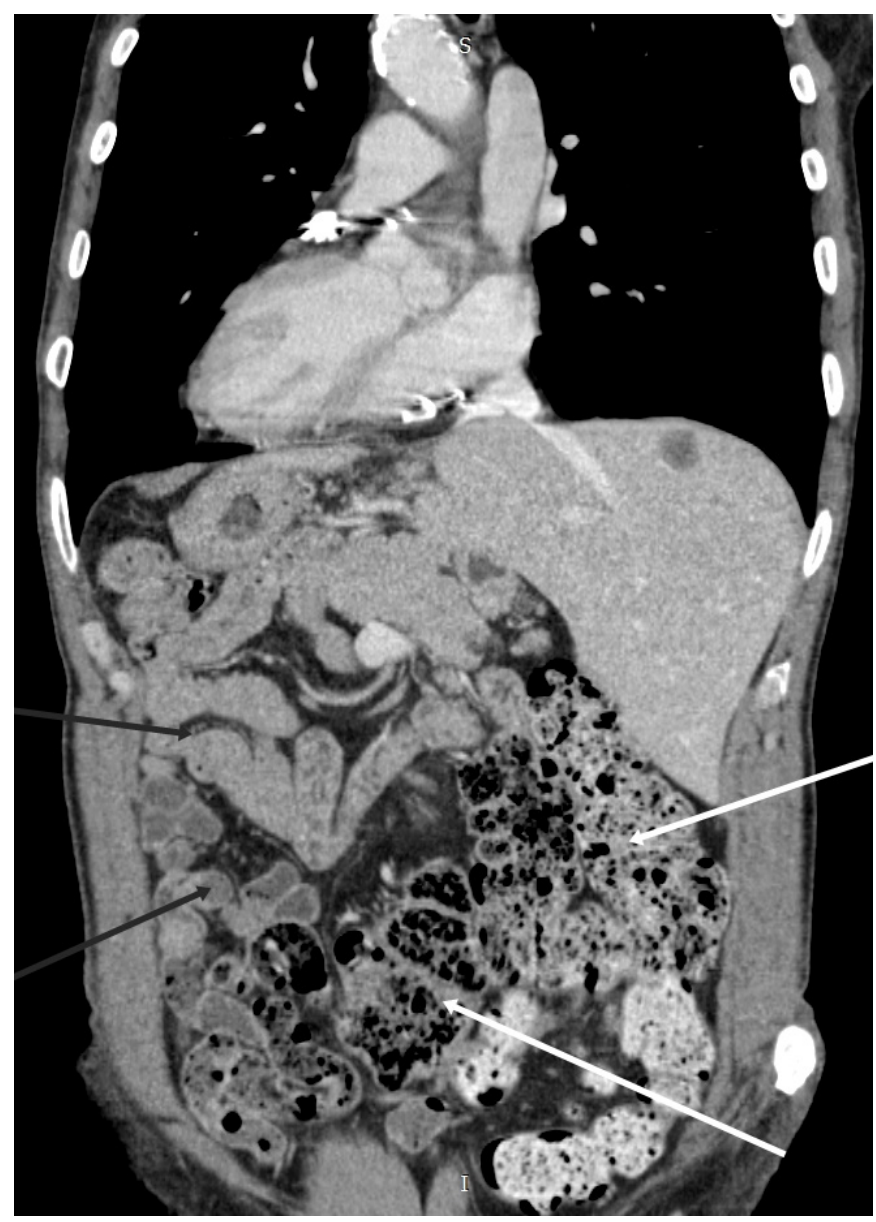

Fig. 1. CT scan image - contrast enhanced MPR coronal view, in portal venous phase, soft tissue window: image depicts dextrocardia, liver with hypovascular metastasis on the left side , large bowel on the left of the midline(white arrows), small bowel on the right of the midline(black arrows) approach as a treatment option. Some authors report the advantage of a left-handed surgeon, but a right-handed specialist can also perform the procedure by changing his position $[4,5,6,7]$. Young Wan Kim et al. reported two cases of SIT. They emphasized the fact that this malformation could predispose to cancer [8]. In the literature some authors reported the occurrence of synchronous and metachronous gastrointestinal malignancies in patients with situs abnormalities (SIT or SA) suggesting the possibility of a connection between unidentified genes responsible for the arrangement of the left-right axis and cancer susceptibility [9]. However, it seems that the incidence of gastrointestinal malignancies in patients with situs abnormalities does not differ significantly from that of malignancies in patients with situs solitus [10].

The anatomical findings represented by bilateral bilobed lungs and polysplenia could suggest a type of SA with double left-sidedness. However, just the right bronchus was hyparterial (bronchial course below the pulmonary artery), whereas the left bronchus was eparterial which strongly suggest the presence of situs inversus. Another anatomical feature was the shape of the atrial appendages, consistent with the anomaly of situs inversus. Polysplenia is also associated with SIT [11]. In the abdomen, the liver was on the left side and on the right side multiple spleens were observed. In most reported cases of polysplenia the agenesis of the body and tail of the pancreas were present. However, in our patient, the pancreas was normally developed, having all three segments [12, 13]. We observed interrupted inferior vena cava with the absence of the hepatic segment and azygos vein continuation. There are case reports of associated interruption of inferior vena cava and SIT, but this is an uncommon condition $[14,15]$. The portal vein had a preduodenal course. There are reported associations among SIT or left isomerism and preduodenal portal vein which sometimes can lead to duodenal obstruction, especially in neonates $[16,17]$. Another uncommon finding in our patient was the aspect of the small and large intestine. The stomach and the duodenum revealed a complete transposition, but the jejunum and the ileum were almost entirely located on the right side of the midline (Figure 1). As a mirror image of the stomach and the duodenum with retroperitoneal fixation of D2, D3 and D4, the jejunum should have been situated on the right side and the ileum on the left side. This was another particular anatomical aspect in our patient, since the ileocecal junction was placed in the front side of the midline, with the rest of the colon on the left side of the abdomen (Figure 3). This finding corresponds to a midgut nonrotation (mesenterium comune). However, in this case, the duodenum was retroperitoneal, with the presence of the ligament of Treitz. The superior mesenteric vein was placed to the right side of the superior mesenteric artery which represents an abnormal position for SIT [18]. This finding is consistent with an inverted rotation of the foregut, whereas the midgut was 

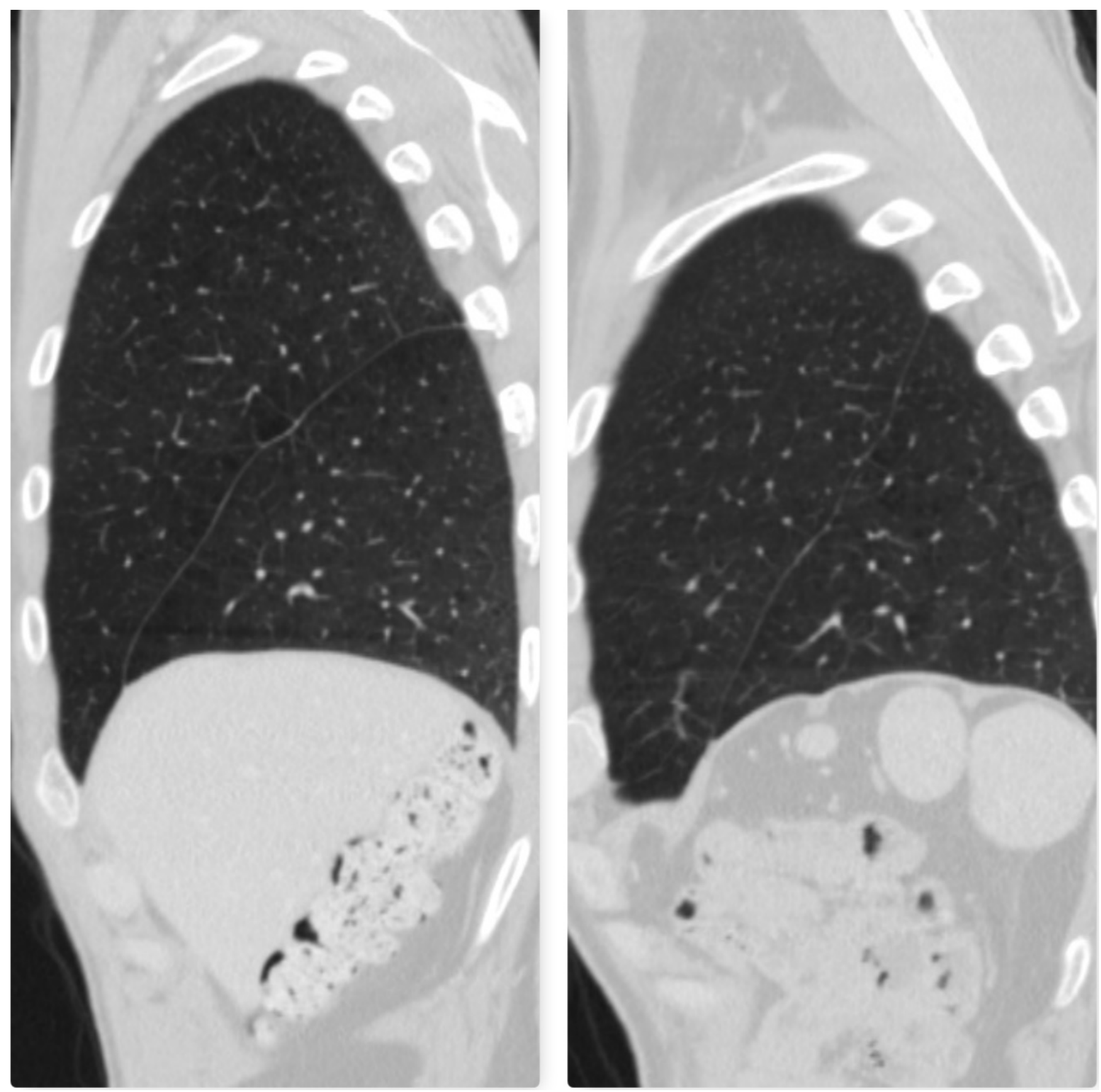

Fig.2. CT scan image - contrast enhanced MPR, sagital view, lung window: on the left, the oblique fissure of the left lung, no horizontal fissure, with liver and colon visualisation below diaphragm; on the right, the oblique fissure of the right lung, with multiple spleens and jejunum below diaphragm

nonrotated. Another case report of ascending colon cancer in a patient with intestinal nonrotation (mesenterium comune) emphasizes the importance of identification of malformations prior to surgery [19]. The presence of distant metastases without involved regional lymphnodes was also an unusual finding. However, tumoral cells were identified, microscopically, in the pericolic fat tissue. The presence of skip metastases involving intermediate colic lymph nodes with normal appearance could be considered. Lymph nodes with homogeneous structure and a short axis diameter up to $3 \mathrm{~mm}$ can contain malignant cells [20]. Brillantino et al., Nakayama et al. and Donner et al. reported cases of colon adenocarcinoma in $\mathrm{T}_{3}$ stage,
$\mathrm{T}_{4 \mathrm{~b}}$ stage respectively, with negative regional lymph nodes in patients with intestinal malrotation [19, 21, 22]. Another 13 case reports of patients with intestinal malrotation and colon cancer revealed that the nonrotation type was the most common finding [21].

\section{Conclusions}

Situs abnormalities do not represent premalignant conditions, the incidence of malignancies in these patients being almost the same as in patients with situs solitus. Preoperative imaging in patients with CRC is necessary for a proper surgical management. The identification of situs abnormality is essential for the right surgical approach, 


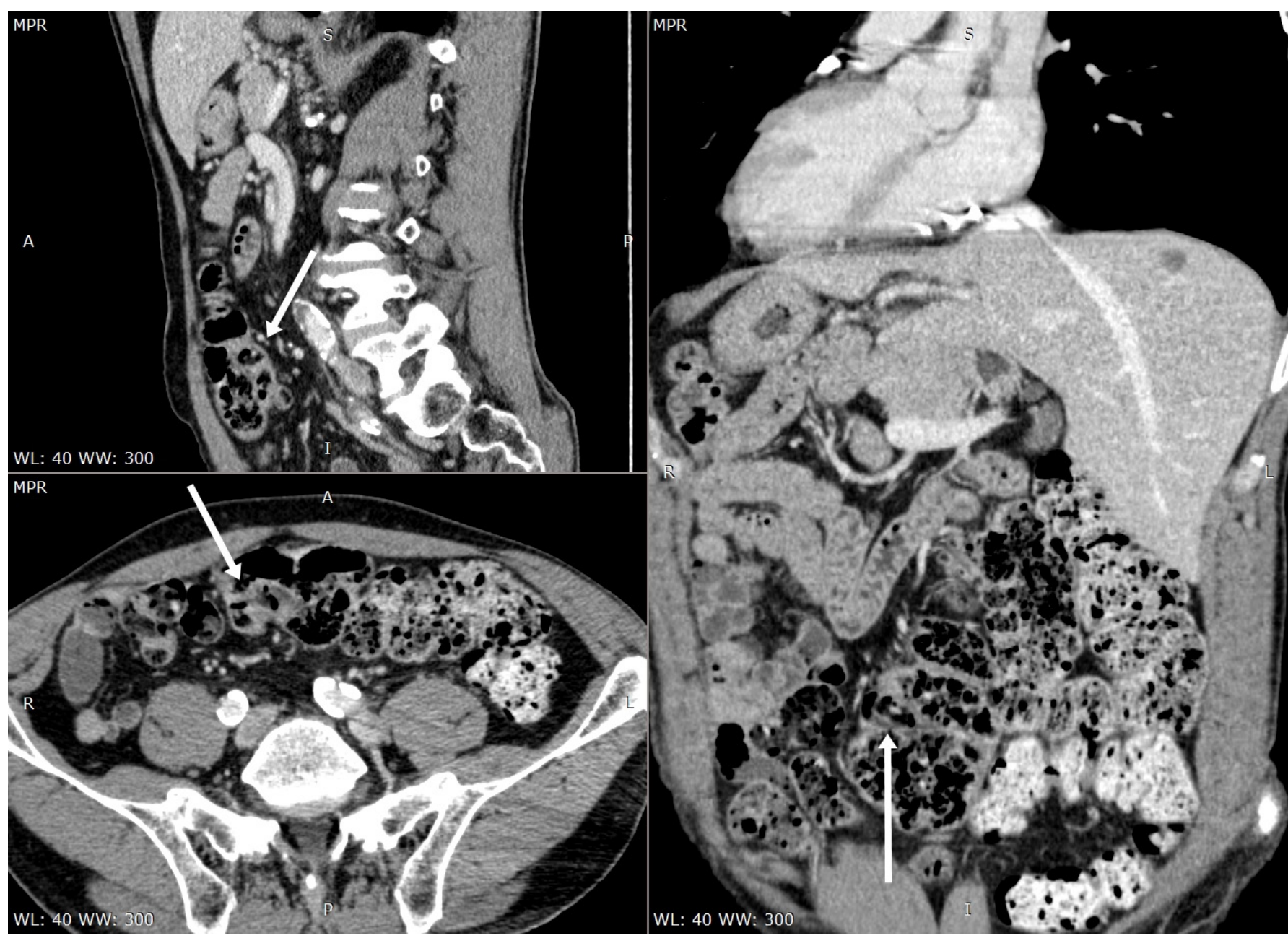

Fig.3. CT scan image - contrast enhanced MPR portal venous phase; white arrows indicate ileocecal junction(upper image sagital view, lower image axial view, right image coronal view)

especially where the laparoscopic technique is considered. Colorectal cancer metastasizing patterns in patients with intestinal malrotation may show unusual findings, further research being necessary. Furthermore, the possibility of metachronous or synchronous tumor incidence should not be overlooked.

\section{Conflict of interest}

None to declare.

\section{References}

1. Siegel RL, Miller KD, Jemal A - Cancer Statistics, 2017. CA Cancer J Clin. 2017; 67 (1):7-30.

2. Applegate KE, Goske MJ, Pierce G, Murphy D - Situs revisited: imaging of the heterotaxy syndrome. Radiographics. 1999;19: 837-852.

3. Basso MP, Christiano AB, Gonçalves-Filho FA, Melo MM, Ronchi LS, Netinho JG - Colorectal cancer and situs inversus totalis : case report. Arq Bras Cir Dig. 2014;27(4):303.

4. Oms LM, Badia JM - Laparoscopic cholecystectomy in situs inversus totalis: the importance of being left-handed. Surg Endosc 2003;17(11):1859-1861.

5. Choi SI, Park SJ, Kang BM, Lee KY, Lee HC, Lee SH - Laparoscopic abdominoperineal resection for rectal cancer in a patient with situs inversus totalis. Surg Laparosc Endosc Percutan Tech. 2011;21(2):e87-90.

6. Davies $H$, Slater $\mathrm{GH}$, Bailey M - Laparoscopic sigmoid colectomy for diverticular disease in a patient with situs inversus. Surg Endosc. 2003;17(1):160-161.

7. Sasaki K, Nozawa H, Kawai K et al - Laparoscopic hemicolectomy for a patient with situs inversus totalis: A case report.Int J Surg Case Rep.
2017; 41: 93-96

8. Kim YW, Ryu H, Kim DS, Kim IY - Double primary malignancies associated with colon cancer in patients with situs inversus totalis: two case reports. World J Surg Oncol. 2011; 9:109

9. Galiatsatos P, Kasprzak L, Chong G, Jass JR, Foulkes WD - Multiple primary malignancies in a patient with situs ambiguus. Clin Genet. 2006; 69:528-31

10. Iwamura T, Shibata N, Haraguchi $Y$ et al - Synchronous double cancer of the stomach and rectum with situs inversus totalis and polysplenia syndrome. J Clin Gastroenterol. 2001; 33:148-53

11. Kayhan A, Lakadamyali $H$, Oommen J, Oto A Polysplenia syndrome accompanied with situs inversus totalis and annular pancreas in an elderly patient. Clin Imaging. 2010; 34(6):472-475

12. Hatayama C, Wells TR - Syndrome of externally bilobed lungs with normal bronchial branch pattern,congenital heart disease, multiple spleens, intestinal malrotation and short pancreas: an apparently hitherto undefined malformation complex. Pediatr Pathol. 1984; 2:127-133.

13. Hadar H, Gadoth N, Herskovitz P, Heifetz M - Short pancreas in polysplenia syndrome. Acta Radiol 1991; 32:299-301.

14. Loukas M, Strike DRG, Tubbs RS, Tarneja N,Shoja MM - An interrupted inferior vena cava in a situs inversus: a case report and review of the literature. Folia Morphol 2009; 68(3):184-187.

15. Del Prete A, Cavaliere C, Di Pietto F, De Ritis R - Situs inversus with levocardia, infrahepatic interruption of the inferior vena cava, and azygos continuation: a case report. Surg Radiol Anat. 2016; 38(3):369-371.

16. D'souza F, Nage A, Bendre P - Preduodenal portal vein with situs inversus totalis causing duodenal obstruction. APSP J Case Rep. 2016; 7(3): 24.

17. Seo HI, Jeon TY, Sim MS, Kim S - Polysplenia syndrome with PDPV detected in adults. World J Gastro- Enterol.2008; 14:6418-6420.

18. Wanjari AK, Deshmukh AJ, Tayde PS, Lonka Y - Midgut Malrotation with Chronic Abdominal Pain. N Am J Med Sci. 2012; 4(4): 196-198.

19. Brillantino A, Marano L, Schettino $M$ et al. - Report of a rare case of colon cancer complicated by anomalies of intestinal rotation and fixation: 
a case report. Cases J. 2009; 2:6555

20. Zhou L, Wang JZ, Wang JT et al. - Correlation analysis of MR/CT on colorectal cancer lymph node metastasis characteristics and prognosis. Eur Rev Pharmacol Sci 2017;21: 1219-1225

21. Nakayama $Y$, Akyama M, Sawatsubashi $Y$ et al - A case of advanced descending colon cancer in an adult patient with intestinal malrotation Case Rep Gastrointest Med 2016; Article ID 3194056, 5 pages

22. Donaire M, Mariadason J, Stephens D, Pillarisety S, Wallack MK Carcinoma of the colon in an adult with intestinal malrotation. Case Rep Surg 2013; Article ID 525081, 4 pages 